\title{
The Population of Fusarium oxysporum f. sp. lactucae in California and Arizona
}

\author{
Kelley R. Paugh and Thomas R. Gordon ${ }^{\dagger}$ \\ Department of Plant Pathology, University of California, Davis, CA 95616
}

Abstract

\begin{abstract}
Fusarium wilt of lettuce, caused by Fusarium oxysporum f. sp. lactucae, is now found in all major lettuce producing regions in California and Arizona. The population structure of $F$. oxysporum $\mathrm{f}$. sp. lactucae in California and Arizona was characterized based on somatic compatibility and sequences of the translation elongation factor $1-\alpha$ gene $(\mathrm{EF}-1 \alpha)$ and rDNA intergenic spacer region (IGS). In this study, 170 isolates were tested for somatic compatibility based on heterokaryon formation, using complementary nitrate nonutilizing (nit) mutants. Five subgroups (A to E) of somatic compatibility group 0300 were identified. Isolates associated with the same subgroup had a strong complementation reaction, whereas reactions between isolates of different subgroups were weak or delayed. An isolate from the first known infestation of Fusarium wilt of lettuce in California was associated with subgroup A, which predominated among isolates in our collection. Isolates representative of each
\end{abstract}

subgroup were confirmed to be associated with race 1 , based on the reaction of differential lettuce cultivars. It is possible that somatic compatibility subgroups B to E of $F$. oxysporum $\mathrm{f}$. sp. lactucae were derived from subgroup A, as a consequence of somatic mutations affecting compatibility. If so, subgroups of $F$. oxysporum $\mathrm{f}$. sp. lactucae may represent an intermediate step in divergence that will lead to clearly separable compatibility groups. Sequences of EF-1 $\alpha$ and IGS were both identical for 58 isolates of $F$. oxysporum f. sp. lactucae that represented all somatic compatibility subgroups and locations from which isolates were obtained, indicating that subgroups were derived from the same clonal lineage (VCG 0300).

Keywords: fungi, Fusarium wilt, Fusarium oxysporum, pathogen diversity, somatic compatibility, vegetables
California and Arizona account for nearly all lettuce produced in the United States (U.S. Department of Agriculture National Agricultural Statistics Service 2019), and all major production areas in both states are now affected by Fusarium wilt. The causal pathogen, Fusarium oxysporum $\mathrm{f}$. sp. lactucae, was first identified as a cause of root rot of lettuce in Japan in 1955 (Matuo and Motohashi 1967). The same pathogen was discovered in a lettuce production field in Fresno County, California, in 1990 and in two nearby fields a year later (Hubbard and Gerik 1993). Thereafter, F. oxysporum f. sp. lactucae was found in six fields near Yuma, Arizona, in 2001 and in 21 additional fields in the same region in 2002 and 2003 (Matheron and Koike 2003; Matheron et al. 2005). Since that time, the pathogen has become established in all major lettuce producing regions on the central coast of California (Scott et al. 2012). Infested soil on farming equipment has likely facilitated movement of the pathogen between fields (Matheron and Koike 2003), with contaminated seed implicated as a means for transoceanic transport (Garibaldi et al. 2004). Fusarium wilt of lettuce now has a worldwide distribution, with documented occurrences in Taiwan (Huang and Lo 1998), Iran (Millani et al. 1999), Italy (Garibaldi et al. 2002), Portugal (Pasquali et al. 2007), Brazil (Ventura and Costa 2008), and Argentina (Malbrán et al. 2014) and reports from five European countries, including the Netherlands (Gilardi et al. 2017a), France (Gilardi et al. 2017b), Belgium (Claerbout et al. 2018), and Ireland and the United Kingdom (Taylor et al. 2019).

\section{${ }^{\dagger}$ Corresponding author: T. R. Gordon; trgordon@ucdavis.edu}

Funding: This work was supported by the U.S. Department of Agriculture National Institute of Food and Agriculture Hatch project CA-D-PPA-6699$\mathrm{H}$, California Leafy Greens Research Board grants, and a University of California Jastro-Shields Research award.

*The $\boldsymbol{e}$-Xtra logo stands for "electronic extra" and indicates that one supplementary table is published online.

The author(s) declare no conflict of interest.

Accepted for publication 6 January 2020.

(C) 2020 The American Phytopathological Society
F. oxysporum f. sp. lactucae has been differentiated into three races in Japan (races 1, 2, and 3) (Fujinaga et al. 2001, 2003; Yamauchi et al. 2004), and race 4 was identified in several European countries (Claerbout et al. 2018; Gilardi et al. 2017a; Taylor et al. 2019). Race 1 has the widest geographic distribution and is the only race to have been identified in North and South America (McCreight et al. 2005; Pasquali et al. 2005). Molecular analyses revealed a polyphyletic origin for $F$. oxysporum $\mathrm{f}$. sp. lactucae (Mbofung et al. 2007), with race 1 of $F$. oxysporum f. sp. lactucae isolates clustering with other formae speciales. Races 1, 2, 3, and 4 are genetically distinct, with each race aligning with a different somatic compatibility group (Fujinaga et al. 2005; Mbofung et al. 2007; Pintore et al. 2017). Past studies have shown that race 1 isolates from Japan belong to the same clonal lineage as isolates from the first infestations in California, and that $F$. oxysporum f. sp. lactucae from California and Arizona are somatically compatible (Ogiso et al. 2002; Pasquali et al. 2005). These findings suggested that a single strain was disseminated across lettuce producing regions in the United States.

To date, global assessments of the $F$. oxysporum f. sp. lactucae population have included only a small number of isolates from California and Arizona (Pasquali et al. 2005). This study was undertaken to more intensively sample the pathogen in these two states in order to determine whether the population is comprised only of the single lineage that has been detected previously. For this purpose, isolates were characterized based on somatic compatibility and DNA sequences of the translation elongation factor $1-\alpha$ gene $(E F-1 \alpha)$ and the intergenic spacer in the nuclear rDNA.

\section{Materials and Methods}

Somatic compatibility. Isolates of $F$. oxysporum f. sp. lactucae used in this study originated from symptomatic lettuce or soil in major lettuce production areas in California and Arizona. Single-spore cultures added to our laboratory culture collection in years 2001 to 2007 ("JCP" code isolates) were obtained as described by Scott et al. (2010a). Isolate JCP043 was a renamed isolate of $F$. oxysporum f. sp. lactucae (HL-2) that was cultured from infected lettuce in the field where Fusarium wilt was discovered in California in 1990 (Hubbard and Gerik 1993). With the exception of GL1515, isolates added to our collection in years 2014 to 2017 ("GL" coded isolates) were received as cultures from Steven Koike of the University of California Cooperative Extension in Monterey County. GL1515 was 
isolated from a taproot of symptomatic lettuce that was grown in a field plot originally infested with $F$. oxysporum f. sp. lactucae (JCP043) as described by Scott et al. (2010b). Isolates, selected to represent the geographic breadth of lettuce production, had a colony morphology typical of $F$. oxysporum f. sp. lactucae (Scott et al. 2010a) and were confirmed to be pathogenic to lettuce using root-dip inoculations as described by Scott et al. (2010a). Single-hyphal tipped cultures were stored on dried filter paper (Gordon and Okamoto 1991).

Cultures of $F$. oxysporum f. sp. lactucae were revived from storage by incubating colonized filter paper on potato dextrose agar (PDA) for up to 12 days and thereafter transferring plugs of colonized agar $\left(1\right.$ to $2 \mathrm{~mm}^{2}$ ) to PDA amended with $4.5 \%$ potassium chlorate. After 7 to 14 days, chlorate-resistant isolates were transferred to Czapek (CZ) medium (Czapek Solution Agar; Difco BD), a minimal medium that includes nitrate as the sole supplemental source of nitrogen. Isolates that grew thinly and lacked aerial mycelium on $\mathrm{CZ}$ medium were identified as nit mutants. Complementary nitrate nonutilizing (nit) phenotypes (nit1 and NITM), differentiated as described by Correll et al. (1987), were obtained for individual isolates of $F$. oxysporum f. sp. lactucae to serve as tester strains. Nit mutants obtained from other isolates were paired with tester strains on CZ. Where aerial mycelium, indicative of wild-type growth, developed along the line of contact between the unknown isolate and one or both of the tester nits, that isolate was assigned to the somatic compatibility group represented by the testers.

The strength of a complementation reaction was scored on an ordinal scale of 0 to 4 , based on the abundance of wild-type growth (Fig. 1). A score of 4 indicated a strong pairing reaction occurring within 7 days that was similar in strength to the pairing reaction observed between strains of a tester pair (i.e., complementary nits of the same isolate). A score of 3 indicated a strong or moderate pairing reaction that was delayed in comparison with the pairing reaction between a tester pair, with robust wild-type growth visible only after 10 to 14 days of incubation. A score of 2 indicated a moderate or weak pairing reaction, with discontinuous wild-type growth that typically appeared after 7 to 10 days. A score of 1 indicated a weak pairing reaction, with a few spots of discontinuous wild-type growth between isolates that typically appeared after 10 to 14 days. Lastly, a score of 0 indicated a negative pairing reaction, with no wildtype growth observed after 14 days. When an isolate reacted weakly with a tester pair (score less than 4), that isolate was paired with a different set of testers. This process was repeated until every isolate was found to have a strong complementation reaction with a tester pair. All weak or negative pairing reactions were confirmed by testing a second nit from the unknown isolate.

Pathogenicity testing. Five isolates of $F$. oxysporum f. sp. lactucae that were each representative of a somatic compatibility subgroup were tested for pathogenicity to differential lettuce cultivars Patriot (no known resistance), Costa Rica No. 4 (resistant to race 1), and Banchu Red Fire (resistant to races 2 and 4) (Gilardi et al. 2017a; McCreight et al. 2005). Sure Shot, a crisphead cultivar known to be susceptible to subgroup A isolates (JCP043 and GL1515) based on previous tests (K. R. Paugh and T. R. Gordon, unpublished data), was included as an additional control. Isolates selected for pathogenicity testing were GL1815 (subgroup A), GL1639 (subgroup B), JCP024 (subgroup C), JCP164 (subgroup D), and JCP047 (subgroup E).

Tests were also conducted by inoculating the susceptible cultivar Sure Shot to determine whether somatic compatibility subgroups were associated with quantitative differences in virulence. Isolates were selected to be representative of each somatic compatibility subgroup and geographic region as follows: subgroup A (JCP043 from Huron, GL1815 from Salinas, GL1487 from Santa Maria, GL1690 from Watsonville, and JCP422 from Yuma), subgroup B (JCP232 from Huron, JCP382 from Salinas, GL1770 from Santa Maria, GL1639 from Watsonville, and GL1541 from Yuma), subgroup C (JCP024 and JCP431 from Yuma), subgroup D (JCP164 from Huron, JCP457 from Salinas, and GL1636 from Watsonville), and subgroup E (JCP047 and JCP058 from Yuma).

Cultures of isolates were prepared by streaking colonized filter paper across PDA in 10-cm-diameter plates and incubating for 9 to
14 days under constant fluorescent light at room temperature. Seeds of each lettuce cultivar were sown in Sunshine Mix \#1 (Sun Gro Horticulture, Agawam, MA) and maintained in a growth chamber with a 14-h photoperiod and day/night temperatures of $23^{\circ} \mathrm{C} / 18^{\circ} \mathrm{C}$, with relative humidity set for $60 \%$. Seedlings at the two- to three- true leaf stage of growth were root-dip inoculated, as described by Scott et al. $\left(2010 \mathrm{~b}\right.$ ), with $5 \times 10^{6}$ spores $/ \mathrm{ml}$ suspensions for pathogenicity testing on differential cultivars or with $1 \times 10^{6}$ spores $/ \mathrm{ml}$ suspensions for virulence testing on Sure Shot. Inoculated seedlings ( $n=6$ to 8 for pathogenicity testing on differentials; $n=10$ for virulence testing) were transplanted into pots ( $9 \mathrm{~cm}$ in diameter and $10 \mathrm{~cm}$ deep) containing Sunshine Mix \#1. Plants were maintained in a growth chamber with day/night temperatures set for $33^{\circ} \mathrm{C} / 23^{\circ} \mathrm{C}$ and a $14-\mathrm{h}$ photoperiod, with relative humidity set for $60 \%$, for 2 weeks. Symptoms of Fusarium wilt were scored on an ordinal scale of 1 to 4 (Scott et al. 2010a). Ratings were assigned as follows: 1, healthy; 2, mild stunting; 3, severe stunting and chlorosis/necrosis; and 4, dead. Plants that died within 7 days after transplanting were assumed to have died independently of the disease and were excluded from the dataset. Inoculations of differentials were conducted twice for each

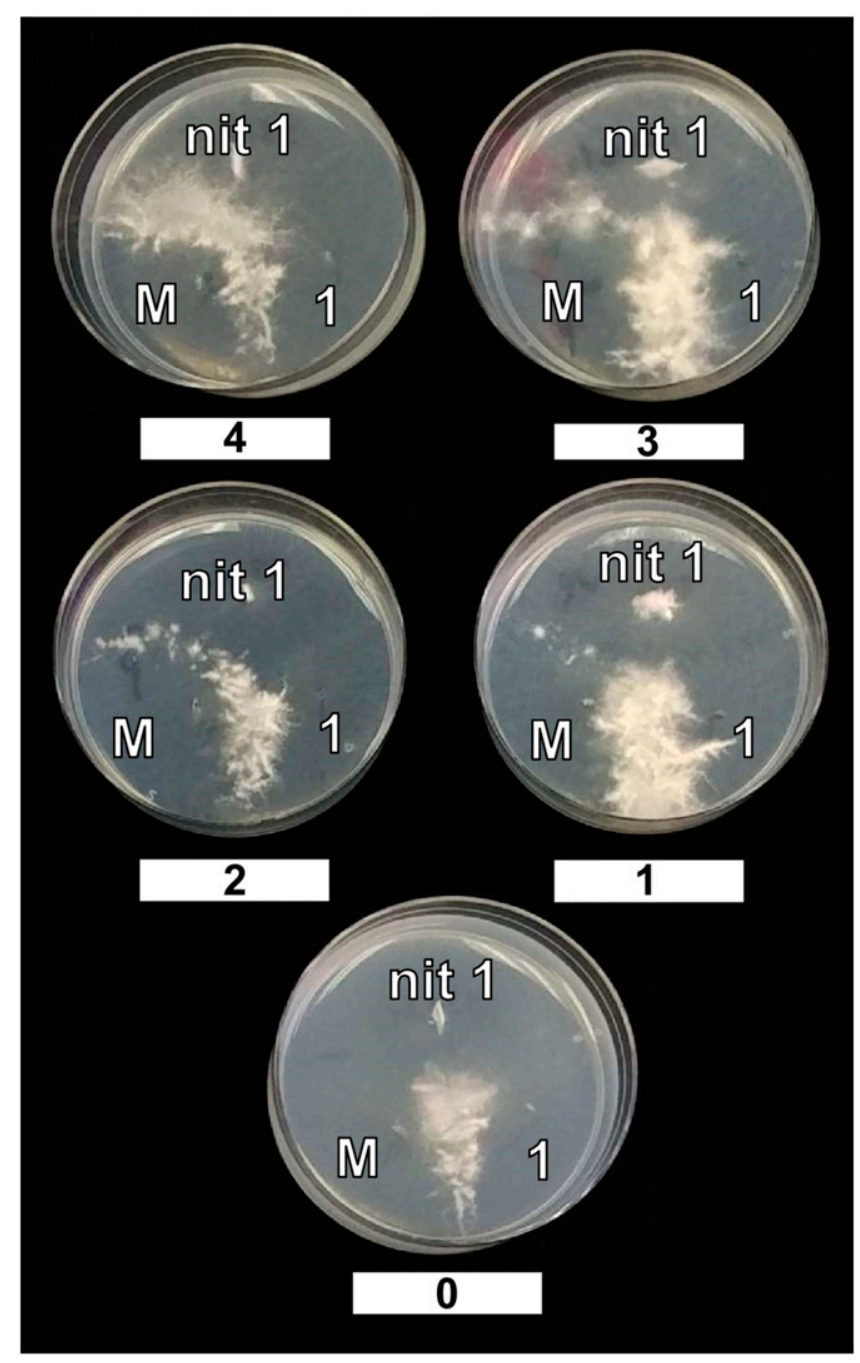

Fig. 1. Scores for strength of complementation between testers and an unknown isolate after 7 days of growth on Czapek (CZ) medium, as indicated by numbers below each plate. $M$ and 1 refer to complementary NITM and nit 1 testers, respectively. nit 1 is derived from an unknown isolate for which somatic compatibility was yet to be determined. Strength of complementation was scored on an ordinal scale of 0 to 4 based on timing and abundance of wild-type growth between isolates on $\mathrm{CZ}$ medium, compared with the positive reaction between testers. Scores (shown below each plate) were assigned as follows: $4=$ abundant wild-type growth within 7 days, 3 = delayed appearance of abundant wild-type growth, 2 = discontinuous wild-type growth, 1 = minimal appearance of discontinuous wild-type growth, and $0=$ no wild-type growth. 
isolate. For virulence tests, each isolate was included in at least two experiments, with additional tests of some isolates based on results of the first two experiments. Statistical analysis of disease severity scores was performed using R version 3.5.0 (R Foundation for Statistical Computing, Vienna, Austria) and RStudio version 1.1.447 (RStudio, Boston, MA). The R software packages rankFD (Brunner et al. 2017; Konietschke et al. 2016) and nparcomp (Konietschke et al. 2015) were employed for rank-based, analysis of variance (ANOVA)-type $P$ tests and nonparametric multiple contrasts of ordinal scores for disease severity.

DNA sequencing. Isolates of $F$. oxysporum $\mathrm{f}$. sp. lactucae were selected to be representative of somatic compatibility subgroups and locations in California and Arizona from which isolates were obtained. Lettuce producing regions were designated as Huron (San Joaquin Valley in proximity to Huron, California), Salinas (in proximity to cities in the Salinas Valley of California: Chualar, Gonzalez, King City, and Salinas), Santa Maria (Santa Maria Valley, California), Watsonville (in proximity to Watsonville, California), and Yuma (in proximity to the cities of Wellton and Yuma, Arizona). At least one and as many as four isolates were selected per field site within a region. Isolates of unknown geographic origin were excluded from DNA sequence analysis.

Isolates of $F$. oxysporum f. sp. lactucae to be tested were grown on PDA for 6 to 10 days. Aerial mycelium was gently scraped from the agar surface and placed in a $1.5-\mathrm{ml}$ tube. DNA extraction of mycelium was carried out using the OmniGen-X PureSpin gDNA Purification Kit (E\&K Scientific Products Inc., Santa Clara, CA) following the manufacturer's instructions, except that vortexing in cell lysis buffer was extended from 10 to $15 \mathrm{~min}$. DNA concentration was measured with an ND-1000 NanoDrop spectrophotometer (NanoDrop Technologies, Wilmington, DE). EF- $1 \alpha$ and the nearly fulllength rDNA intergenic spacer region (IGS) were amplified using PCR in a thermal cycler (T100 Thermal Cycler; Bio-Rad Laboratories Inc., Hercules, CA). The EF- $1 \alpha$ locus was amplified with the primer pair EF1/EF2 using the procedure described by O'Donnell et al. (1998). The partial IGS region was amplified with primer pairs iNL11/CNSa and iCNS11/NLa using the procedure described by O'Donnell et al. (2009). PCR products were visualized with electrophoresis at 100 to $120 \mathrm{~V}$ in $1 \%$ agarose gels stained with GelRed Nucleic Acid Gel Stain (Biotium, Fremont, CA) in $1 \times$ Tris-acetate EDTA buffer and UV transillumination with AlphaImager 2200 (Alpha Innotech Corporation, San Leandro, CA).

PCR products were purified using the Invitrogen PureLink PCR Purification Kit (Thermo Fisher Scientific, Waltham, MA), according to the manufacturer's instructions. DNA concentration and purity of PCR products were assessed using the ND-1000 NanoDrop spectrophotometer. Purified PCR products were sent to Quintara Biosciences (South San Francisco, CA) for Sanger sequencing of the EF- $1 \alpha$ gene and IGS region. Raw reads of forward and reverse sequences were cleaned up and aligned with the MUSCLE algorithm (Edgar 2004) in MEGA version 7.0.14 (Kumar et al. 2016) to obtain a consensus sequence. DNA sequences of EF-1 $\alpha$ and IGS for five isolates that were each representative of a somatic compatibility subgroup were deposited into GenBank. Accession codes for the EF-1 $\alpha$ sequences are MH412701 for GL1815 (subgroup A), MH412702 for GL1639 (subgroup B), MH412703 for JCP024 (subgroup C), MH412704 for JCP164 (subgroup D), and MH412705 for JCP047 (subgroup E). Accession codes for IGS sequences are MH412706 for GL1815 (subgroup A), MH412707 for GL1639 (subgroup B), MH412708 for JCP024 (subgroup C), MH412709 for JCP164 (subgroup D), and MH412710 for JCP047 (subgroup E).

\section{Results}

Somatic compatibility. We tested 145 isolates of $F$. oxysporum $\mathrm{f}$. sp. lactucae from infected lettuce plants and 25 isolates from soil for somatic compatibility (Supplementary Table S1). There were 117 isolates from California, of which 55 were from Salinas, 20 from Watsonville, 16 from the Santa Maria Valley, nine from Huron, 16 from unknown locations in California, and one reisolated from lettuce grown at University of California, Davis. Fifty-three isolates were from the Yuma region of Arizona. Nit1 mutants were generated for 164 isolates and NITM mutants were generated for 85 isolates. Both nit 1 and NITM mutants were obtained for 79 isolates, whereas only a single nit mutant was obtained for 22 isolates.

Testers for isolate GL1815 were paired with a nit 1 or NITM of every other isolate and scored for strength of complementation. If the first pairing failed to result in a strong complementation reaction (score of less than 4), a different mutant of the same isolate was paired with GL1815 testers. The same procedure was carried out for the remaining isolates until each isolate was resolved to pair strongly with a tester strain obtained from one of the following isolates: GL1815 (subgroup A), GL1639 (subgroup B), JCP024 (subgroup C), JCP164 (subgroup D), or JCP047 (subgroup E) (Fig. 2). The only exception was JCP404, for which the strongest interisolate pairing reaction (score $=3$ ) was with testers representing subgroups $\mathrm{A}(\mathrm{JCP} 271)$ and C (JCP012). Isolates that paired strongly $($ score $=4)$ with a tester representing a subgroup were assigned to that subgroup. Isolates in the same subgroup all had a strong positive reaction with one another. Most isolates were also compatible with isolates of a different somatic compatibility subgroup, but the reaction was either delayed or weak (score of less than 4). No isolates were found to complement strongly (score $=4$ ) with testers of more than one subgroup.

Isolates assigned to somatic compatibility subgroups originated from 50 lettuce production fields, 35 in California and 15 in Arizona (Fig. 3; Table 1). There was no apparent difference in the frequency

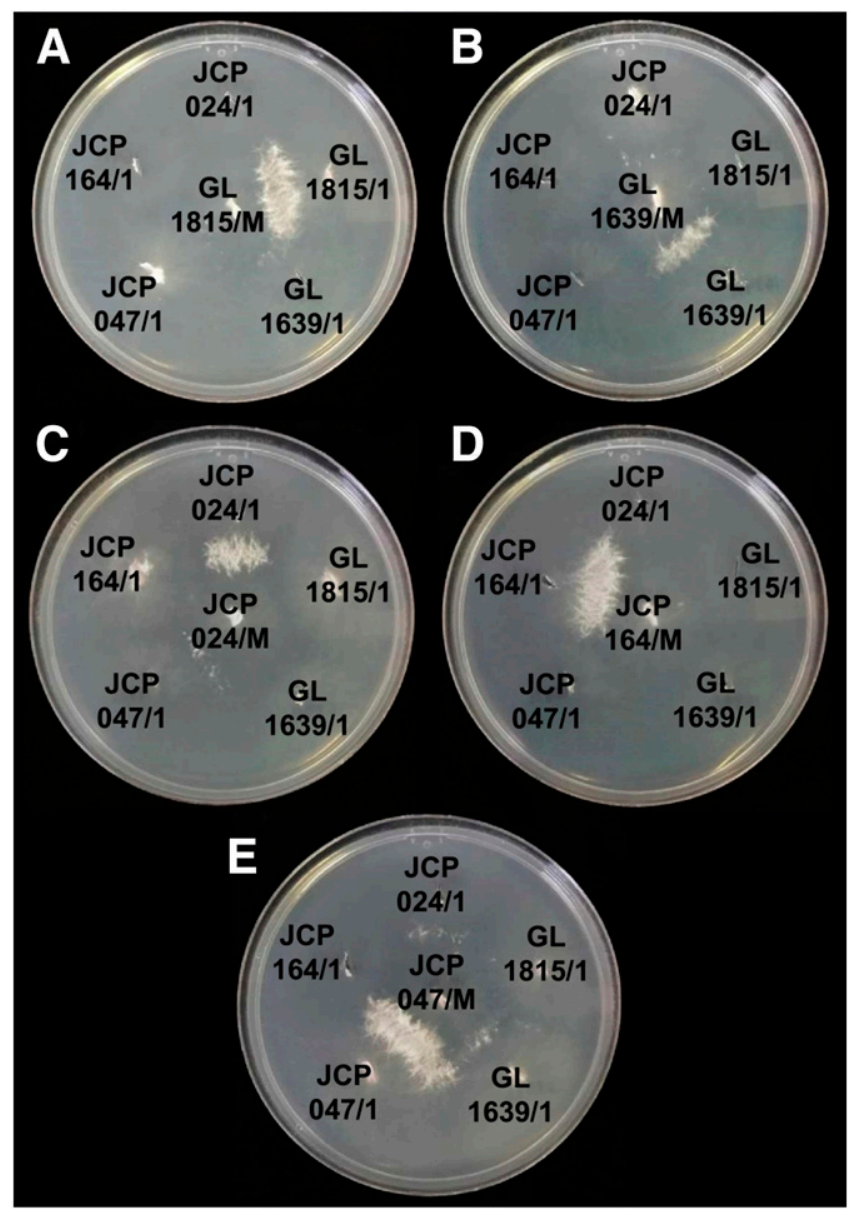

Fig. 2. Representation of somatic compatibility subgroups as demonstrated by a strong complementation reaction between isolates of the same subgroup and a weaker reaction among isolates of different subgroups. Plates show complementation of nit mutants (NITM and nit1) placed $2.5 \mathrm{~cm}$ apart on Czapek medium after 7 days. NITM mutants are located at the center of each plate, representing each subgroup from $A$ to $E$, as indicated in the upper left corner above each plate. Each NITM is surrounded by a nit1 of each subgroup: A, GL1815, B, GL1639, C, JCP024, D, JCP164, and E, JCP047. 
of somatic compatibility subgroups recovered from lettuce compared with soil. Subgroups A, B, and C accounted for $95 \%$ of the isolates, with 106 isolates in subgroup A, 34 isolates in subgroup B, and 22 isolates in subgroup C. Isolate JCP043, from the first infestation discovered in California, was placed in subgroup A. Subgroups D and E included four and three isolates, respectively. Isolates associated with subgroups $\mathrm{A}$ and $\mathrm{B}$ were found in every lettuce producing region in California and Arizona. Subgroups C and E were present in Arizona but not in California, whereas isolates associated with subgroup D were not in Arizona but were found in three regions in California (Huron, Salinas, and Watsonville). Most isolates in subgroup $\mathrm{C}$ were

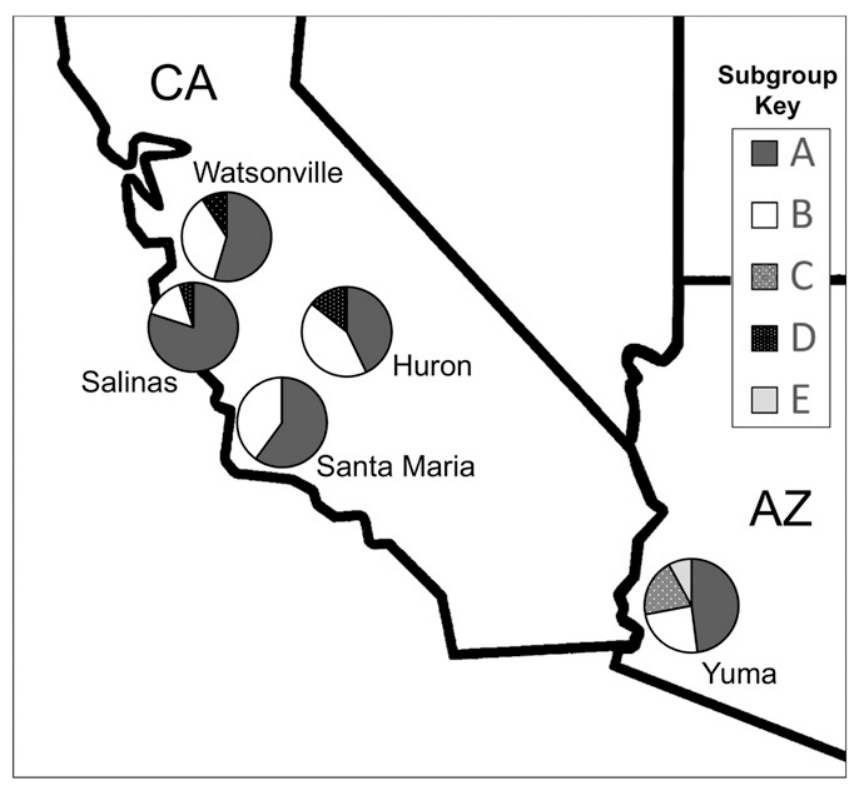

Fig. 3. Map representing the distribution of somatic compatibility subgroups (A to $E$ ) of F. oxysporum f. sp. lactucae in California (CA) and Arizona (AZ) by region: Huron (San Joaquin Valley in proximity to Huron, California), Salinas (in proximity to cities in the Salinas Valley of California: Chualar, Gonzalez, King City, and Salinas), Santa Maria (Santa Maria Valley, California), Watsonville (in proximity to Watsonville, California), and Yuma (in proximity to the cities of Wellton and Yuma, Arizona). Pie charts represent the proportion of field sites with a somatic compatibility subgroup within a region.

Table 1. Occurrence of somatic compatibility subgroups of Fusarium oxysporum f. sp. lactucae by region

\begin{tabular}{|c|c|c|c|c|c|c|c|}
\hline \multirow[b]{2}{*}{ State } & \multirow[b]{2}{*}{ Region $^{\mathbf{a}}$} & \multirow{2}{*}{$\begin{array}{l}\text { Total field } \\
\text { sites }(n)^{\mathbf{b}}\end{array}$} & \multicolumn{5}{|c|}{$\begin{array}{c}\text { Isolates in a } \\
\text { compatibility subgroup } \\
(n)^{\mathbf{c}} \\
\end{array}$} \\
\hline & & & $\mathbf{A}$ & B & $\mathbf{C}$ & $\mathbf{D}$ & $\mathbf{E}$ \\
\hline California & Huron & 5 & 4 & 3 & 0 & 2 & 0 \\
\hline California & Salinas & 16 & 50 & 4 & 0 & 1 & 0 \\
\hline California & Watsonville & 8 & 12 & 7 & 0 & 1 & 0 \\
\hline California & Santa Maria Valley & 6 & 12 & 4 & 0 & 0 & 0 \\
\hline Arizona & Yuma & 15 & 15 & 12 & 22 & 0 & 3 \\
\hline Total $^{\mathrm{d}}$ & & 50 & 93 & 30 & 22 & 4 & 3 \\
\hline
\end{tabular}

a Lettuce producing regions in California and Arizona from which isolates were obtained: Huron (San Joaquin Valley in proximity to Huron, California), Salinas (in proximity to cities in the Salinas Valley of California: Chualar, Gonzalez, King City, and Salinas), Santa Maria Valley (in California), Watsonville (in proximity to Watsonville, California), and Yuma (in proximity to the cities of Wellton and Yuma, Arizona).

b Total number of lettuce producing fields within a region, where $F$. oxysporum f. sp. lactucae was isolated from either lettuce or soil.

c Number of isolates associated with a somatic compatibility subgroup from the region indicated. Isolate JCP404 (from the Yuma region) could not be placed in a compatibility subgroup and therefore was not included in total value.

${ }^{\mathrm{d}}$ Isolates of unknown geographic origin (12 in subgroup A; four in subgroup B) and an isolate from diseased lettuce at University of California, Davis (one in subgroup A) were excluded from total values. sourced from a single field in Wellton, Arizona, but were also found in four other fields in the Yuma region. Subgroup E was represented by isolates from only two field sites in Arizona. Isolates of different subgroups were occasionally recovered from the same field. For example, isolates associated with subgroups A, B, and D were obtained from a single field in the Huron region of California.

Pathogenicity testing. Five isolates, one representative of each somatic compatibility subgroup, all induced Fusarium wilt symptoms in Patriot and Banchu Red Fire but not Costa Rica No. 4. These results indicate that isolates of each somatic compatibility subgroup are all race 1 .

Seventeen isolates were tested for quantitative differences in virulence on a susceptible lettuce cultivar (Table 2). Mean disease severity scores, pooled across experiments and isolates, were $3.0 \pm 0.1$ for subgroup A, $2.9 \pm 0.1$ for subgroup B, $3.1 \pm 0.1$ for subgroup C, $3.1 \pm$ 0.1 for subgroup $\mathrm{D}$, and $2.9 \pm 0.1$ for subgroup $\mathrm{E}$. ANOVA-type statistics (ATS) indicated that effects of experiment $(P<0.001)$ and the interaction of experiment and subgroup $(P=0.006)$ were significant, whereas the effect of subgroup alone was not $(P=0.307)$. Because of a significant interaction term, the effect of subgroup was analyzed separately per experiment as a source of variation in virulence. The effect of subgroup was significant in the first experiment $(P=$ $0.013)$ but not in the second experiment $(P=0.477)$.

For most isolates, there was no significant difference in severity scores between the two experiments $(P \geq 0.166)$. Four isolates for which results differed between the two tests were included in two additional inoculation experiments. Two-way ATS indicated that the effect of isolate was significant $(P=0.011)$, whereas effects of experiment and the interaction of experiment and isolate were not $(P \geq$ $0.089)$. Disease severity scores were only significantly different $(P$ $<0.05$ ) between two isolates (JCP043 and JCP058) within this subset. Overall, there were no consistent differences in disease severity scores between isolates in subgroups.

DNA sequences. The EF- $1 \alpha$ locus and the IGS region were sequenced for 58 isolates of $F$. oxysporum f. sp. lactucae: 39 from California (15 from the Salinas region, 10 from the Watsonville region, seven from the Huron region, six from the Santa Maria Valley region, and one reisolated from lettuce at University of California, Davis) and 19 from the Yuma region of Arizona. Thirty-one isolates were associated with subgroup A, 12 with subgroup B, eight with

Table 2. Virulence of isolates associated with five somatic compatibility subgroups of Fusarium oxysporum f. sp. lactucae

\begin{tabular}{lcccccc}
\hline & & \multicolumn{5}{c}{ Disease severity score $^{\mathbf{b}}$} \\
\cline { 3 - 6 } Subgroup $^{\mathbf{a}}$ & Isolate & Trial 1 & Trial 2 & Trial 3 & Trial 4 & Grand $^{\mathbf{c}}$ \\
\hline A & JCP043 & $2.4 \pm 0.3$ & $3.5 \pm 0.2$ & $3.4 \pm 0.2$ & $3.0 \pm 0.4$ & $3.0 \pm 0.2$ \\
A & JCP422 & $3.3 \pm 0.3$ & $3.2 \pm 0.2$ & - & - & $3.2 \pm 0.2$ \\
A & GL1487 & $3.1 \pm 0.4$ & $2.8 \pm 0.2$ & - & - & $3.0 \pm 0.2$ \\
A & GL1690 & $2.6 \pm 0.4$ & $3.1 \pm 0.2$ & - & - & $2.8 \pm 0.2$ \\
A & GL1815 & $2.9 \pm 0.4$ & $3.5 \pm 0.2$ & - & - & $3.2 \pm 0.2$ \\
B & JCP232 & $3.3 \pm 0.3$ & $2.8 \pm 0.3$ & - & - & $3.1 \pm 0.2$ \\
B & JCP382 & $3.3 \pm 0.3$ & $3.2 \pm 0.2$ & - & - & $3.2 \pm 0.2$ \\
B & GL1541 & $2.7 \pm 0.3$ & $3.1 \pm 0.2$ & - & - & $2.9 \pm 0.2$ \\
B & GL1639 & $1.8 \pm 0.3$ & $3.2 \pm 0.2$ & $3.0 \pm 0.1$ & $1.9 \pm 0.4$ & $2.5 \pm 0.2$ \\
B & GL1770 & $3.3 \pm 0.3$ & $3.1 \pm 0.3$ & - & - & $3.2 \pm 0.2$ \\
C & JCP024 & $3.9 \pm 0.1$ & $2.6 \pm 0.2$ & $3.0 \pm 0.2$ & $2.8 \pm 0.3$ & $3.1 \pm 0.1$ \\
C & JCP431 & $3.3 \pm 0.3$ & $3.3 \pm 0.1$ & - & - & $3.3 \pm 0.2$ \\
D & JCP164 & $2.7 \pm 0.3$ & $2.6 \pm 0.3$ & - & - & $2.7 \pm 0.2$ \\
D & JCP457 & $3.6 \pm 0.3$ & $2.9 \pm 0.2$ & - & - & $3.3 \pm 0.2$ \\
D & GL1636 & $3.3 \pm 0.2$ & $3.2 \pm 0.3$ & - & - & $3.3 \pm 0.2$ \\
E & JCP047 & $3.4 \pm 0.3$ & $3.2 \pm 0.3$ & - & - & $3.3 \pm 0.2$ \\
E & JCP058 & $3.5 \pm 0.2$ & $2.8 \pm 0.2$ & $1.9 \pm 0.2$ & $2.5 \pm 0.4$ & $2.7 \pm 0.2$ \\
\hline
\end{tabular}

${ }^{a}$ Isolates were representative of five somatic compatibility subgroups of $F$. oxysporum f. sp. lactucae.

${ }^{b}$ Mean disease severity scores \pm SE in repeated experiments (trials 1 to 4 ) for isolates tested on the susceptible lettuce cultivar, Sure Shot. Dashes indicate that additional experiments were not performed for the given isolate.

${ }^{\mathrm{c}}$ Mean disease severity scores \pm SE based on data pooled across experiments for isolates tested on the susceptible lettuce cultivar, Sure Shot. 
subgroup C, four with subgroup D, and two with subgroup E. One isolate was not associated with a subgroup (JCP404). Final sequence lengths were 687 to $695 \mathrm{bp}$ for EF-1 $\alpha$ and 2,210 to 2,309 bp for IGS. All EF-1 $\alpha$ sequences were identical among isolates. Likewise, IGS sequences were identical among isolates. Because of the absence of polymorphisms in EF- $1 \alpha$ and IGS sequences, there was no further analysis of sequence data. EF- $1 \alpha$ and IGS sequences of isolates used in this study are identical to GenBank accessions DQ837657 and DQ831873, respectively, of $F$. oxysporum f. sp. lactucae isolates that were included in phylogenetic analyses performed by Mbofung et al. (2007) and Gilardi et al. (2017a).

\section{Discussion}

Five subgroups (A to E) within a single somatic compatibility group were newly identified among 170 isolates of $F$. oxysporum f. sp. lactucae from California and Arizona. Two isolates, JCP043 (subgroup A) and JCP024 (subgroup C), were previously assigned to VCG 0300 (Pasquali et al. 2005); hence, all isolates used in our study are associated with this compatibility group. Isolates representative of each somatic compatibility subgroup were tested on differential cultivars and confirmed to belong to race 1 . The results of this study are therefore consistent with the presence of only one clonal lineage of $F$. oxysporum f. sp. lactucae in California and Arizona.

Somatic compatibility subgroups document diversity in the population of $F$. oxysporum $\mathrm{f}$. sp. lactucae throughout lettuce production areas in California and Arizona. Because our sample includes isolates collected over an extended period of time and is not balanced across locations, it constitutes only an overview of the population. Assessing the significance of local differences in diversity of subgroups would require a hierarchical sampling scheme, as described by Gordon et al. (1992) for a study of $F$. oxysporum populations in native and cultivated soils.

Somatic compatibility subgroups have also been reported in $F$. oxysporum f. sp. radicis-lycopersici (Katan et al. 1991) and F. oxysporum f. sp. melonis (Katan et al. 1994). In these formae speciales, slow and often weak or discontinuous wild-type growth was observed in pairings between subgroups, compared with strong reactions within subgroups, which is similar to how subgroups are defined in this study. The differential strength of pairing reactions is presumably related to the frequency of hyphal fusions between paired strains, which in turn determines the abundance of reduced nitrogen available to support growth. The genetic basis for this variation is not known but could be attributable to somatic mutations that negatively affect compatibility. This implies that compatibility has a quantitative aspect and is not simply a qualitative trait determined by a well-defined set of loci (Leslie 1993). Quantitative differences in somatic compatibility in $F$. oxysporum have been reported previously (Gordon and Okamoto 1991).

An isolate from the first known infestation of Fusarium wilt of lettuce in California was associated with subgroup A, which predominated among isolates in our collection. It is therefore possible that other subgroups of $F$. oxysporum $\mathrm{f}$. sp. lactucae were derived from subgroup A. If so, subgroups of $F$. oxysporum $\mathrm{f}$. sp. lactucae may represent an intermediate step in divergence that will lead to clearly separable compatibility groups. A more advanced stage in this process has been reported for $F$. circinatum. In this case, multiple compatibility groups were associated with the same clonal lineage (Wikler and Gordon 2000), and experimentation showed that one could be derived from another through a spontaneous change that was deemed likely attributable to a somatic mutation (Petersen and Gordon 2005).

Previous studies have reported very little genetic diversity between isolates associated with a given race of $F$. oxysporum $\mathrm{f}$. sp. lactucae (Fujinaga et al. 2005; Mbofung et al. 2007). Consistent with these findings, 58 isolates of $F$. oxysporum $\mathrm{f}$. sp. lactucae examined in this study revealed all IGS and EF- $1 \alpha$ sequences to be identical. Thus, these two loci were not informative for inferring relationships among F. oxysporum f. sp. lactucae isolates from California and Arizona. A more sensitive measure of variation in this population was provided by differences in somatic compatibility. These differences suggest that the population of $F$. oxysporum $\mathrm{f}$. sp. lactucae has undergone structural changes since its introduction to the United States.
Our studies revealed no consistent association between somatic compatibility subgroups and differences in virulence. This does not preclude the possibility that virulence differences exist between or within somatic compatibility subgroups of $F$. oxysporum $\mathrm{f}$. sp. lactucae, but detecting such differences may require a test with greater resolving power than a root-dip assay. In any case, the observed quantitative differences in somatic compatibility are suggestive of relatively frequent somatic mutations, some of which may lead to the emergence of new pathotypes.

\section{Acknowledgments}

We gratefully acknowledge contributions of Jeness Scott and Steven Koike to the culture collection, seeds donated by Jim McCreight and supplied courtesy of Ryan McMillen of Rijk Zwaan, technical advice from Ana Pastrana León and Abigail Stack, and technical assistance from Zion Congrave-Wilson, Lauren Downing, Aimee Hopkins, Hannah Houts, and Arpad Karsai.

\section{Literature Cited}

Brunner, E., Konietschke, F., Pauly, M., and Puri, M. L. 2017. Rank-based procedures in factorial designs: Hypotheses about non-parametric treatment effects. J. R. Stat. Soc. B 79:1463-1485.

Claerbout, J., Venneman, S., Vandevelde, I., Decombel, A., Bleyaert, P., Volckaert, A., Neukermans, J., and Hofte, M. 2018. First report of Fusarium oxysporum f. sp. lactucae race 4 on lettuce in Belgium. Plant Dis. 102:1037.

Correll, J. C., Klittich, C., and Leslie, J. 1987. Nitrate nonutilizing mutants of Fusarium oxysporum and their use in vegetative compatibility tests. Phytopathology 77:1640-1646.

Edgar, R. C. 2004. MUSCLE: Multiple sequence alignment with high accuracy and high throughput. Nucleic Acids Res. 32:1792-1797.

Fujinaga, M., Ogiso, H., Shinohara, H., Tsushima, S., Nishimura, N., Togawa, M., Saito, H., and Nozue, M. 2005. Phylogenetic relationships between the lettuce root rot pathogen Fusarium oxysporum $\mathrm{f}$. sp. lactucae races 1, 2, and 3 based on the sequence of the intergenic spacer region of its ribosomal DNA. J. Gen. Plant Pathol. 71:402-407.

Fujinaga, M., Ogiso, H., Tsuchiya, N., and Saito, H. 2001. Physiological specialization of Fusarium oxysporum f. sp. lactucae, a causal organism of Fusarium root rot of crisp head lettuce in Japan. J. Gen. Plant Pathol. 67:205-206.

Fujinaga, M., Ogiso, H., Tuchiya, N., Saito, H., Yamanaka, S., Nozue, M., and Kojima, M. 2003. Race 3, a new race of Fusarium oxysporum f. sp. lactucae determined by a differential system with commercial cultivars. J. Gen. Plant Pathol. 69:23-28.

Garibaldi, A., Gilardi, G., and Gullino, M. 2002. First report of Fusarium oxysporum on lettuce in Europe. Plant Dis. 86:1052.

Garibaldi, A., Gilardi, G., and Gullino, M. L. 2004. Seed transmission of Fusarium oxysporum f. sp. lactucae. Phytoparasitica 32:61-65.

Gilardi, G., Franco Ortega, S., Rijswick, P., Ortu, G., Gullino, M. L., and Garibaldi, A. 2017a. A new race of Fusarium oxysporum f. sp. lactucae of lettuce. Plant Pathol. 66:677-688.

Gilardi, G., Pons, C., Gard, B., Franco-Ortega, S., and Gullino, M. L. 2017b. Presence of Fusarium wilt, incited by Fusarium oxysporum f. sp. lactucae, on lettuce in France. Plant Dis. 101:1053.

Gordon, T. R., and Okamoto, D. 1991. Vegetative compatibility groupings in a local population of Fusarium oxysporum. Can. J. Bot. 69:168-172.

Gordon, T. R., Okamoto, D., and Milgroom, M. G. 1992. The structure and interrelationship of fungal populations in native and cultivated soil. Mol. Ecol. 1:241-249.

Huang, J., and Lo, C. 1998. Wilt of lettuce caused by Fusarium oxysporum in Taiwan. Plant Pathol. Bull. 7:150-153.

Hubbard, J., and Gerik, J. 1993. New wilt disease of lettuce incited by Fusarium oxysporum f. sp. lactucum forma specialis nov. Plant Dis. 77:750-755.

Katan, T., Katan, J., Gordon, T. R., and Pozniak, D. 1994. Physiologic races and vegetative compatibility groups of Fusarium oxysporum f. sp. melonis in Israel. Phytopathology 84:153-157.

Katan, T., Zamir, D., Sarfatti, M., and Katan, J. 1991. Vegetative compatibility groups and subgroups in Fusarium oxysporum f. sp. radicis-lycopersici. Phytopathology 81:255-262.

Konietschke, F., Friedrich, S., Brunner, E., and Pauly, M. 2016. RankFD: Rankbased tests for general factorial designs, version 0.0 .1 . https://rdrr.io/cran/ rankFD/

Konietschke, F., Placzek, M., Schaarschmidt, F., and Hothorn, L. A. 2015. nparcomp: An R software package for nonparametric multiple comparisons and simultaneous confidence intervals, version 2.6.0. R Project for Statistical Computing, Vienna, Austria. https://cran.r-project.org

Kumar, S., Stecher, G., and Tamura, K. 2016. MEGA7: Molecular evolutionary genetics analysis version 7.0 for bigger datasets. Mol. Biol. Evol. 33: $1870-1874$

Leslie, J. F. 1993. Fungal vegetative compatibility. Annu. Rev. Phytopathol. 31: 127-150.

Malbrán, I., Mourelos, C., Mitidieri, M., Ronco, B., and Lori, G. 2014. Fusarium wilt of lettuce caused by Fusarium oxysporum f. sp. lactucae in Argentina. Plan Dis. 98:1281. 
Matheron, M. E., and Koike, S. T. 2003. First report of Fusarium wilt of lettuce caused by Fusarium oxysporum f. sp. lactucae in Arizona. Plant Dis. 87:1265.

Matheron, M. E., McCreight, J. D., and Tickes, B. R. 2005. Effect of planting date, cultivar, and stage of plant development on incidence of Fusarium wilt of lettuce in desert production fields. Plant Dis. 89:565-570.

Matuo, T., and Motohashi, S. 1967. On Fusarium oxysporum f. sp. lactucae n. f. causing root rot of lettuce. Trans. Mycol. Soc. Jpn. 32:13-15.

Mbofung, G. Y., Hong, S. G., and Pryor, B. M. 2007. Phylogeny of Fusarium oxysporum f. sp. lactucae inferred from mitochondrial small subunit, elongation factor 1- $\alpha$, and nuclear ribosomal intergenic spacer sequence data. Phytopathology 97:87-98.

McCreight, J. D., Matheron, M. E., Tickes, B. R., and Platts, B. 2005. Fusarium wilt race 1 on lettuce. HortScience 40:529-531.

Millani, M., Etebarian, H., and Alizadeh, A. 1999. Occurrence of Fusarium wilt of lettuce in Shahre-Ray, Varamin and Karaj areas. Iran J. Plant Pathol. 35:44-45.

O’Donnell, K., Gueidan, C., Sink, S., Johnston, P. R., Crous, P. W., Glenn, A., Riley, R., Zitomer, N. C., Colyer, P., and Waalwijk, C. 2009. A two-locus DNA sequence database for typing plant and human pathogens within the Fusarium oxysporum species complex. Fungal Genet. Biol. 46:936-948.

O’Donnell, K., Kistler, H. C., Cigelnik, E., and Ploetz, R. C. 1998. Multiple evolutionary origins of the fungus causing Panama disease of banana: Concordant evidence from nuclear and mitochondrial gene genealogies. Proc. Nat. Acad. Sci. 95:2044-2049.

Ogiso, H., Fujinaga, M., Saito, H., Takehara, T., and Yamanaka, S. 2002. Physiological races and vegetative compatibility groups of Fusarium oxysporum f. sp. lactucae isolated from crisphead lettuce in Japan. J. Gen. Plant Pathol. 68: 292-299.

Pasquali, M., Dematheis, F., Gilardi, G., Gullino, M. L., and Garibaldi, A. 2005. Vegetative compatibility groups of Fusarium oxysporum f. sp. lactucae from lettuce. Plant Dis. 89:237-240.
Pasquali, M., Dematheis, F., Gullino, M. L., and Garibaldi, A. 2007. Identification of race 1 of Fusarium oxysporum f. sp. lactucae on lettuce by inter-retrotransposon sequence-characterized amplified region technique. Phytopathology 97:987-996.

Petersen, J. C., and Gordon, T. R. 2005. Spontaneous changes in vegetative compatibility in the ascomycete Gibberella circinata. Phytopathology 95:583.

Pintore, I., Gilardi, G., Gullino, M., and Garibaldi, A. 2017. Analysis of vegetative compatibility groups of Italian and Dutch isolates of Fusarium oxysporum $\mathrm{f}$. sp. lactucae. J. Riv. Patol. Veg. 99:517-521.

Scott, J., Gordon, T. R., Kirkpatrick, S., Koike, S., Matheron, M., Ochoa, O., Truco, M., and Michelmore, R. 2012. Crop rotation and genetic resistance reduce risk of damage from Fusarium wilt in lettuce. Calif. Agric. 66:20-24.

Scott, J. C., Gordon, T. R., Shaw, D. V., and Koike, S. T. 2010a. Effect of temperature on severity of Fusarium wilt of lettuce caused by Fusarium oxysporum f. sp. lactucae. Plant Dis. 94:13-17.

Scott, J. C., Kirkpatrick, S. C., and Gordon, T. R. 2010b. Variation in susceptibility of lettuce cultivars to Fusarium wilt caused by Fusarium oxysporum f. sp. lactucae. Plant Pathol. 59:139-146.

Taylor, A., Jackson, A. C., and Clarkson, J. P. 2019. First report of Fusarium oxysporum f. sp. lactucae race 4 causing lettuce wilt in England and Ireland. Plant Dis. 103:1033.

U.S. Department of Agriculture National Agricultural Statistics Service. 2019 Vegetables 2018 Summary (March 2019). https://usda.library.cornell.edu/

Ventura, J., and Costa, H. 2008. Fusarium wilt caused by Fusarium oxysporum on lettuce in Espirito Santo, Brazil. Plant Dis. 92:976.

Wikler, K., and Gordon, T. R. 2000. An initial assessment of genetic relationships among populations of Fusarium circinatum in different parts of the world. Can J. Bot. 78:709-717.

Yamauchi, N., Shimazu, J., Satou, M., Horiuchi, S., and Shirakawa, T. 2004. Physiological races and vegetative compatibility groups of butterhead lettuce isolates of Fusarium oxysporum f. sp. lactucae in Japan. J. Gen. Plant Pathol. 70:308-313. 